\title{
Atomic-scale observation of the deformation and failure of diamonds by in-situ double-tilt mechanical testing transmission electron microscope holder
}

\author{
Yizhi Zhang ${ }^{1,2 \dagger}$, Yeqiang $\mathrm{Bu}^{1 \dagger}$, Junquan Huang ${ }^{3}$, Tianye $\mathrm{Jin}^{3}$, Anmin $\mathrm{Nie}^{3^{*}}$, Hongtao Wang ${ }^{1,2^{*}}$ and \\ Yongjun Tian ${ }^{3 *}$
}

\begin{abstract}
In-situ transmission electron microscopy (TEM) has been demonstrated to be a powerful method in resolving challenging problems such as interactions among various defects. To take advantage of the atomic resolution of advanced TEMs, a compact five-degree-of-freedom nanomanipulator was integrated with an indenter that was made of nanotwinned diamonds, for both the in-situ mechanical testing and double tilting of TEM samples. As a demonstration, in-situ bending tests were performed on the $\langle 111\rangle,\langle 110\rangle$ and $\langle 100\rangle$ single-crystal diamond needles. The tests revealed the $\{111\}$ cleavage to be the dominant failure mode. The in-situ indentation on a diamond nanoplate led to curved cracks consisting of nanometer-scale steps, which were identified to be atomic flat $\{111\}$ facets. The atomic-scale observation of the deformation and failure of diamonds demonstrated the stability of the entire system and the durability of the indenter. We expect that more delicate research can be carried out by means of this holder in the near future, including in-situ stimulation, atomic characterization, and tomography.
\end{abstract}

Keywords: mechanical testing holder, double tilt, diamond, in situ TEM, tomography

\section{INTRODUCTION}

The most advanced transmission electron microscopes (TEMs) have pushed their spatial resolution to $\sim 0.5 \AA$ since their invention [1-3]. Such unparalleled resolution renders a TEM a unique tool in characterizing the atomic structures of crystalline materials in great depth [4]. More attention has been paid to in-situ TEM that provides detailed information about microstructural evolution instead of still post-mortem images [5-8]. The dynamic pictures enrich the understanding of the interactions among various defects, which are important in unveiling the mechanisms of macroscopic phenomena [7-10]. Currently, it is still a challenge to combine an atomicscale observation with an in-situ stimulus, especially under great mechanical loads [11]. The reasons for this are twofold. For the operation side, mechanical loading always leads to the displacements of atoms, lowering the imaging resolution. As a compromise, a TEM sample can be deformed incrementally in a step-wise manner. Then atomic resolution images can be taken in each step when the load is held still. This requires that the testing system has high stiffness and excellent mechanical stability. For the hardware side, atomic resolution TEM imaging requires the crystal orientation of a TEM sample to be aligned to the electron beam direction. To satisfy this condition, tilting samples in two orthogonal directions must be used. One tilting function is built-in with TEM, and it involves rotating a sample around the holder axis, i.e., $\alpha$-tilt. The other tilting function, known as $\beta$-tilt, is generally driven by an electrical motor inside a TEM holder. These designs leave nearly no space for mechanical load actuators. Most commercially available in-situ mechanical testing TEM holders do not provide the $\beta$-tilt option [12]. Other types of stimuli such as electrical biasing and heating can be achieved by integrating electrodes and heating wires into microelectromechanical system (MEMS) chips as sample supports. The $\beta$-tilt

\footnotetext{
${ }^{1}$ Center for X-Mechanics, Zhejiang University, Hangzhou 310027, China

${ }^{2}$ Institute of Applied Mechanics, Zhejiang University, Hangzhou 310027, China

${ }^{3}$ Center for High Pressure Science, State Key Laboratory of Metastable Materials Science and Technology, Yanshan University, Qinhuangdao 066004, China

These authors contributed equally to this work.

* Corresponding authors (emails: anmin@ysu.edu.cn (Nie A); htw@zju.edu.cn (Wang H); fhcl@ysu.edu.cn (Tian Y))
} 
function can thus be realized by installing MEMS chips into a double-tilt TEM holder [13]. However, MEMS chips are prohibitively expensive, discouraging the wide acceptance of in-situ TEM.

Recently, diamond has been proposed as a promising electronic material to replace silicon because of the property of an elastic strain-tunable wide bandgap [1417]. However, the brittleness of diamond at room temperature limits the possibility of strain engineering. Though recent studies have demonstrated the superior elastic deformation capability of diamond $[18,19]$, it is still unclear how the deformation and failure behaviors are related to strain [18]. Because diamond is the hardest material known in nature, it is a great challenge to perform in-situ TEM experiments on its yielding and failure. The group at the Center for X-Mechanics [20] has developed the X-Nano TEM holder integrated with a fourdegree-of-freedom nano-manipulator. All the movements of the four degrees of freedom are precisely driven by the built-in piezoelectric actuators, minimizing artifacts due to the vibration and drifting of the TEM stage. The TEM sample can be freely translated in space and rotated around the holder axis for $360^{\circ}$. The usage for TEM tomography applications has been demonstrated in characterizing the three-dimensional (3D) dislocation network in Si [20]. In this work, we added the $\beta$-tilt function and an indenter made of high-hardness and high-toughness nanotwinned diamond (NTD) [21] to the $\mathrm{X}$-Nano holder, achieving the atomic-scale observation of the deformation and failure of diamonds. The $\{111\}$ cleavage was identified to be the dominant failure mechanism. We hope that extensive research can be carried out with this holder in the near future, including in-situ stimulation, atomic characterization, and tomography.

\section{EXPERIMENTAL SECTION}

\section{Design of the in-situ double-tilt mechanical testing TEM holder}

The in-situ double-tilt mechanical testing TEM holder, called the X-Mech holder for abbreviation, was based on the X-Nano manipulator (Fig. 1a), which was developed by our group and dedicated to TEM tomography applications [20]. It had four degrees of freedom, including three-directional positioning plus $360^{\circ}$ self-rotation around the holder axis. All of the movements of the four degrees of freedom were driven by precise built-in piezoelectric actuators. The positioning accuracy was less than $0.1 \mathrm{~nm}$ in all three directions, and the rotation angle was calibrated within $\pm 0.05^{\circ}$. More information is supplied in Ref. [20]. To enable atomic resolution characterization, the fifth degree of freedom, i.e., the $\beta$-tilt, was achieved by replacing the fixed sample base (inset of Fig. 1a) with a movable sample base (Fig. 1b). Fig. 1c shows the exploded view of the mechanical design with the different parts labeled by capital letters. Part A is the movable sample base that was assembled by the inertial slider mechanism. The actuators in parts B and C consisted of the stack of an in-plane polarized piezoelectric plate and a smooth alumina plate with a total thickness of
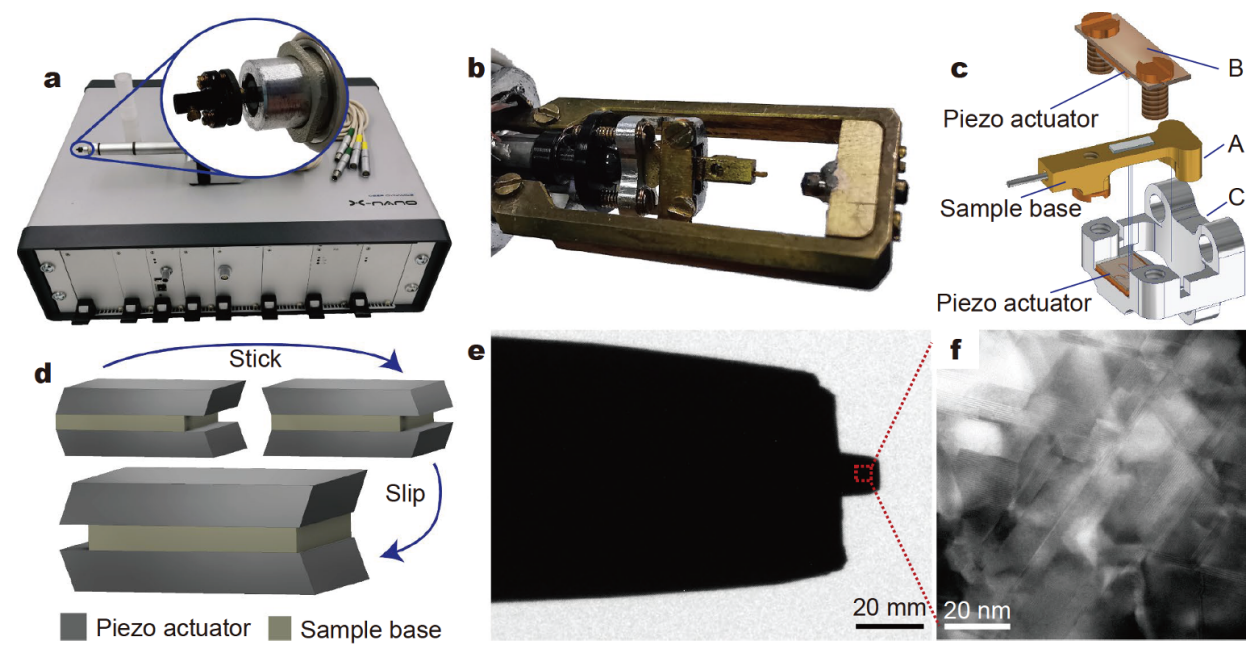

Figure 1 (a) The X-Nano TEM holder [20]. (b) The front end of the in-situ double-tilt mechanical testing TEM holder. (c) The exploded view of the mechanical design. Different parts are indicated by the capital letters. Two thin plate piezo actuators were fixed on parts B and C. (d) A schematic showing the stick-slip process. (e) A low-magnification TEM image of the flat-head indenter made of NTD. (f) The bright field TEM image of the NTD. 
$0.25 \mathrm{~mm}$. By applying a sawtooth waveform for the voltage, the piezoelectric plate repeated the deformation of a slow increase of an in-plane shear deformation followed by a fast recovery. In one period, the sample base moved as it "stuck to" the piezoelectric plate during the shear deformation due to the friction force upon a proper pressure from the cover. The sample stayed nearly still when the recovering process was fast enough. A schematic is shown in Fig. 1d. It was noted that the shear deformation only led to a translational displacement of the alumina plate. In part $\mathrm{C}$, a curved trench was machined to guide the sample base and change its translation to rotation around the center, where the TEM sample was located. In this way, the height change of the TEM sample was minimized after the $\beta$-tilt. In one stick-slip step, the $\beta$-tilt was measured to be around $0.005^{\circ}$, which was accurate enough to align the TEM samples to a specific crystal orientation. Given the sawtooth waveform frequency of $200 \mathrm{~Hz}$, a $\beta$-tilt of $1^{\circ}$ could be achieved in $1 \mathrm{~s}$. The $\beta$-tilt range was $\pm 8^{\circ}$ due to the limitation of the guiding trench in part $\mathrm{C}$.

For the compact design of the X-Nano manipulator and the movable sample base, an indenter could be seamlessly integrated into the front-end chip of the TEM holder (Fig. 1e). Inset of Fig. 1e shows the TEM image of the indenter, which was made of NTD. The NTD microstructure (Fig. 1f) was characterized by high-density nanoscale $\{111\}$ coherent twins with boundaries known to be strong barriers for dislocation motion. The Vickers hardness and the fracture toughness of the NTD were about $200 \mathrm{GPa}$ and $10 \mathrm{MPa} \mathrm{m}^{0.5}$ [21], approximately two times the values for natural diamond. By employing this superhard material, nearly all materials, including NTD, could be deformed under compression without causing the failure of the indenter.

Indenter fabrication and preparation of the diamond samples The NTD indenter was fabricated by a pulsed femtosecond-laser (PHAROS 10W with Yb: KGW based system) machining. The laser system could provide a pulse duration of $\sim 220 \mathrm{fs}$ at $343 \mathrm{~nm}$ with a maximum energy of $202 \mu \mathrm{J}$. We used natural type Ia diamond and NTD to fabricate diamond nanoneedles, diamond nanopillars, diamond nanoplates, and NTD pillars via focused ion beam (FIB) milling with a current of $0.5 \mathrm{nA}$ under a voltage of $30 \mathrm{kV}$. Residual amorphous carbon on the FIBmilled crystalline surface was then removed with Argon plasma cleaning by gradually decreasing the voltages from 2.0 to $1.5,1.0$ and finally $0.8 \mathrm{kV}$.

Mechanical testing experiments were carried out using the X-Mech TEM holder. The samples were placed on the tip of a $200-\mu \mathrm{m}$ tungsten wire that was fixed on the movable sample base. All of the samples were compressed against the indenter in a step-by-step displacement/ loading process. Bending/buckling occurred for the diamond nanoneedles with high aspect ratios when the applied load reached Euler's critical point. For the NTD pillars, the deformation was pure compression before crack initiation. For the single-crystal diamond nanoplate samples, the stress state was complex under indentation.

\section{Finite element method (FEM) analyses}

An FEM analysis was employed to calculate the strain distribution of the bending diamond nanoneedles using the ABAQUS software package (Dassault Systèmes Simulia Corp.). The calculation details can be found in our previous study [18].

\section{First-principle simulations}

The theoretical elastic instability limit of a single-crystal diamond was explored by the Vienna ab initio simulation package based on density functional theory with the plane-wave pseudopotential approach. The ultra-soft pseudo-potentials of the Rappe-Rabe-Kaxiras-Joannopoulos (RRKJ) type [22] were employed within the local density approximation (LDA) for the exchange-correlation potentials. For the plane-wave basis set, the cutoff energy for the plane waves was $800 \mathrm{eV}$ for the simulations of a bulk diamond crystal. The self-consistency error was set to be $10^{-8}$ Ry. The Monkhorst-Pack [23] $k$-point mesh of $3 \times 3 \times 3$ was chosen for the structural optimization, and a denser grid of $10 \times 10 \times 10$ was applied in calculating the charge density contour. The residual force components on each atom were set to be less than $10^{-3} \mathrm{eV} / \mathrm{Bohr}$ for structural optimization. The uniaxial tension was simulated by increasing the stain in a stepwise manner. In each step, an incremental strain of $0.1 \%$ was applied to the unit cell by adjusting the atomic positions according to the affine transformation given by the strain tensor. The size of the transformed cell was fixed in the tensile direction in the following structure, while it was free to be adjusted in the other two orthogonal directions. In this way, the nontensile stress components could be reduced to less than $0.02 \mathrm{GPa}$, and the non-tensile stress components were negligible compared with the tensile stress.

\section{RESULTS AND DISCUSSION}

\section{Compression of NTD}

NTD has attracted intense attention due to its promising 
mechanical properties and thermal stability [21]. However, due to technical limitations for testing, the deformability of NTD remains unknown so far. Here, for the first time, we experimentally investigated the deformability of an NTD pillar by using the X-Mech holder inside TEM. A typical TEM image of an NTD nanopillar that was fabricated by FIB is shown in Fig. 2a, presenting a non-homogeneous contrast inside the nanopillar induced by NTD nanograins. The length of the NTD nanopillar was measured to be about $361 \mathrm{~nm}$, and its side edge was straight before compression. The corresponding selected area electron diffraction (SAED) pattern containing the (111), (022), and (113) diffraction rings of the diamond further demonstrated the polycrystalline feature of the NTD nanopillar. Fig. 2c-g show the in-situ compression process of the NTD nanopillar. Remarkably, the NTD nanopillar was gradually deformed by a stepwise loading, indicating a high loading force supplied by the holder. After moving the NTD nanopillar backward, as shown in Fig. $2 \mathrm{~h}$, there was an obvious shape change of the NTD nanopillar before and after the compression, indicating the occurrence of the permanent plastic deformation of the NTD nanopillar. According to the length of the nanopillar $(340 \mathrm{~nm})$ after the compression, a 5.8\% plastic deformation was achieved in the NTD nanopillars. This was the first time such a high plastic deformation in diamond was achieved at room temperature. This result confirmed that the X-Mech holder could supply a load high enough to make the hardest material yield inside the TEM. By taking advantage of this mechanical testing holder, we also observed room-temperature plasticity mediated by dislocation in a single-crystal diamond during the uniaxial compression [24,25]. Interestingly, the recent first principle calculations showed that a large ductility could be achieved in the diamond under the coexisting large shear and compressive stress [26]. It seemed that the stress state played an important role in activating the plasticity of the diamond. However, the underlying mechanism of plastic deformation of NTD still requires deep investigation in the future [27].

\section{Bending of single-crystal diamonds}

Diamond is considered a next-generation ideal widebandgap semiconductor due to its high breakdown field, thermal conductivity, and electron mobility. Theoretical investigations have indicated that introducing elastic strain is an effective way of engineering the bandgap of diamond [14]. However, the elastic strain in bulk diamond is always very limited due to premature fracturing. The study of the fracture mechanism of diamond is the key to improve the diamond's elastic limit and illuminate the fabrication of novel semiconductors. In this research, we employed the X-Mech holder to study the elastic bending and fracture behaviors of diamond nanoneedles in order to achieve great elastic deformation. The $\langle 111\rangle$, $\langle 110\rangle$ and $\langle 100\rangle$-oriented diamond nanoneedles were precisely driven against the ultra-hard NTD indenter. Significant elastic deformation occurred in those diamond nanoneedles (as shown in Fig. 3a1-c1), which would be difficult to approach in bulk diamonds. The FEM analysis was performed to quantitatively estimate the strain of the bending nanoneedles (Fig. 3a2-c2). The maximum tensile strains of the bending nanoneedles in Fig. $3 \mathrm{a} 1-\mathrm{c} 1$ were estimated to be as high as $8.6 \%, 5.2 \%$, and $11.2 \%$. Fig. 3a3-c3 show the sharp fracture surfaces of the $\langle 111\rangle,\langle 110\rangle$ and $\langle 100\rangle$-oriented diamond nanoneedles, respectively. Combined with the corresponding atomically-resolution images and SAED patterns, shown in Fig. 3a4-c4, we could determine that those fracture surfaces were composed of atomic-flat $\{111\}$ cleavage facets. Such a "clean" fracture was closely related to the smooth surface and the paucity of inner defects of those diamond nanoneedles. It should be stated that all of the bending diamond nanoneedles studied here deformed elastically, and there was no evidence showing that the stress-induced phase transition occurred. Some artifacts
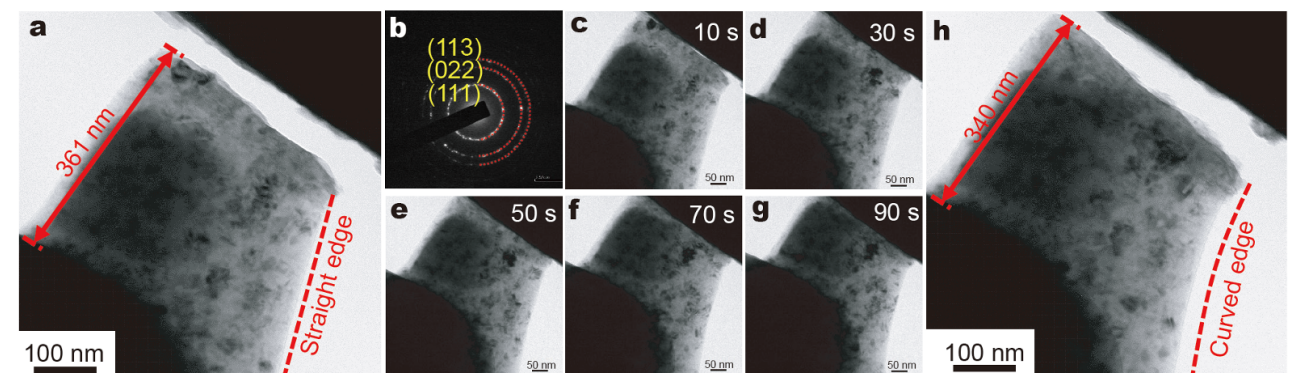

Figure 2 In situ compression of the NTD pillar. (a) TEM image of an NTD pillar before compression. (b) SAED pattern of the NTD pillar. (c-g) Time-lapse morphology evolution of the NTD pillar during in situ compression. (h) TEM image of the NTD pillar after compression. 

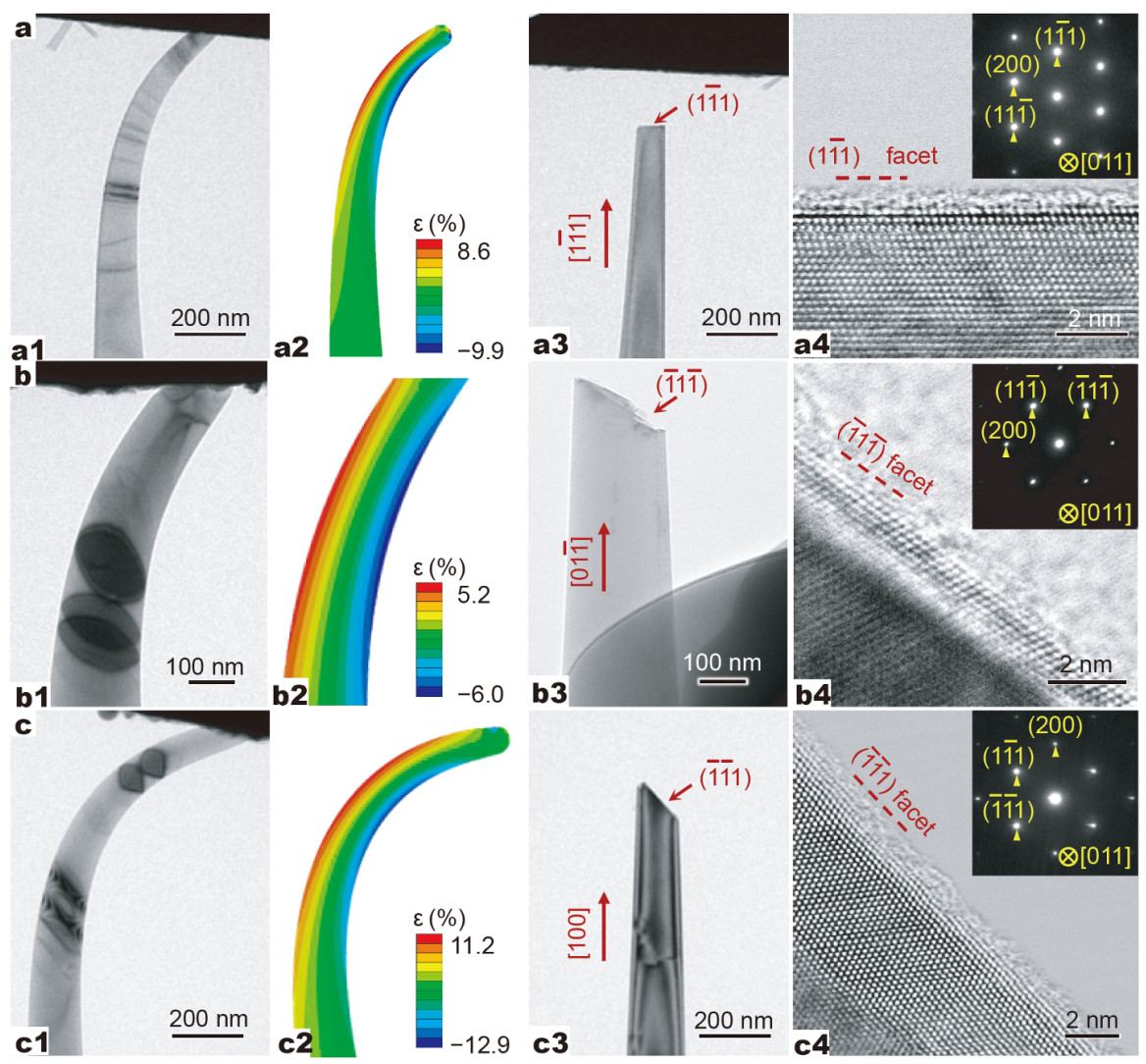

Figure 3 In situ bending of the single-crystal diamond nanoneedles. (a) Bending and fracture of the $\langle 111\rangle$-oriented diamond nanoneedles. (a1) Critical shape before fracture; (a2) corresponding FEM simulation results; (a3) morphology after fracturing; (a4) atomic resolution characterization of the fractured surface. The inset of (a4) shows the corresponding SAED. (b, c) Bending and the fracturing of the $\langle 110\rangle$ - and $\langle 100\rangle$-oriented diamond nanoneedles, respectively.

on the fractured surfaces in Fig. 3a4-c4 were probably caused by the delocalization effect of high-resolution TEM imaging or the residual amorphous layer produced during the sample preparation.

The crack morphology could be clearly delineated by taking advantage of the built-in $360^{\circ}$ rotation function of the X-Mech holder. Fig. 4 shows the bright-field TEM images of a deflected crack produced in a bending diamond nanopillar at various viewing angles. Fig. 4a reveals that the crack was initiated from a corner, which generally led to stress concentration. Based on the contrast from the trace, the crack propagation could be separated into four stages, which are labeled as I to IV in Fig. 4a. In stage I, the crack had a long and flat surface. Based on the SAED shown in Fig. 4a, we could tell that stage I was the consequence of the $\{111\}$ cleavage. The crack was deflected in stages II and IV. In stage II, the crack had a curved geometry. After an $\alpha$-tilt of $45^{\circ}$ (Fig. $4 \mathrm{~b}$ ), this segment showed a sawtooth pattern with an angle of $\sim 110^{\circ}$ between adjacent facets, which was composed of two $\{111\}$ cleavage facets. At other view angles (Fig. 4c-f), the trace had an elliptical shape. By combining the edgeon geometry in Fig. 4a, we could tell that this trace was due to the intersection between a plane and a cylinder.

\section{In-situ observation of the crack propagation in diamond}

Based on the above results, it was identified that the $\{111\}$ cleavage was the dominant mechanism in the fracture of a single-crystal diamond. For the cylindrical geometry, the elastic energy of the whole system drove the crack to propagate quickly, which could only just be captured by the TEM camera for the slow scan rate of $2 \mathrm{~Hz}$. Additionally, the maximum crack extension was in the order of the cylinder diameter. The maximum crack extension quickly broke the structure once the crack was nucleated. To minimize the energy release in the crack tip and to maximize the crack propagation length, the geometry was specially designed to be a thin plate for in-situ observation of the crack propagation (Fig. 5a). For this purpose, we used a conical shape indenter with a tip radius of 

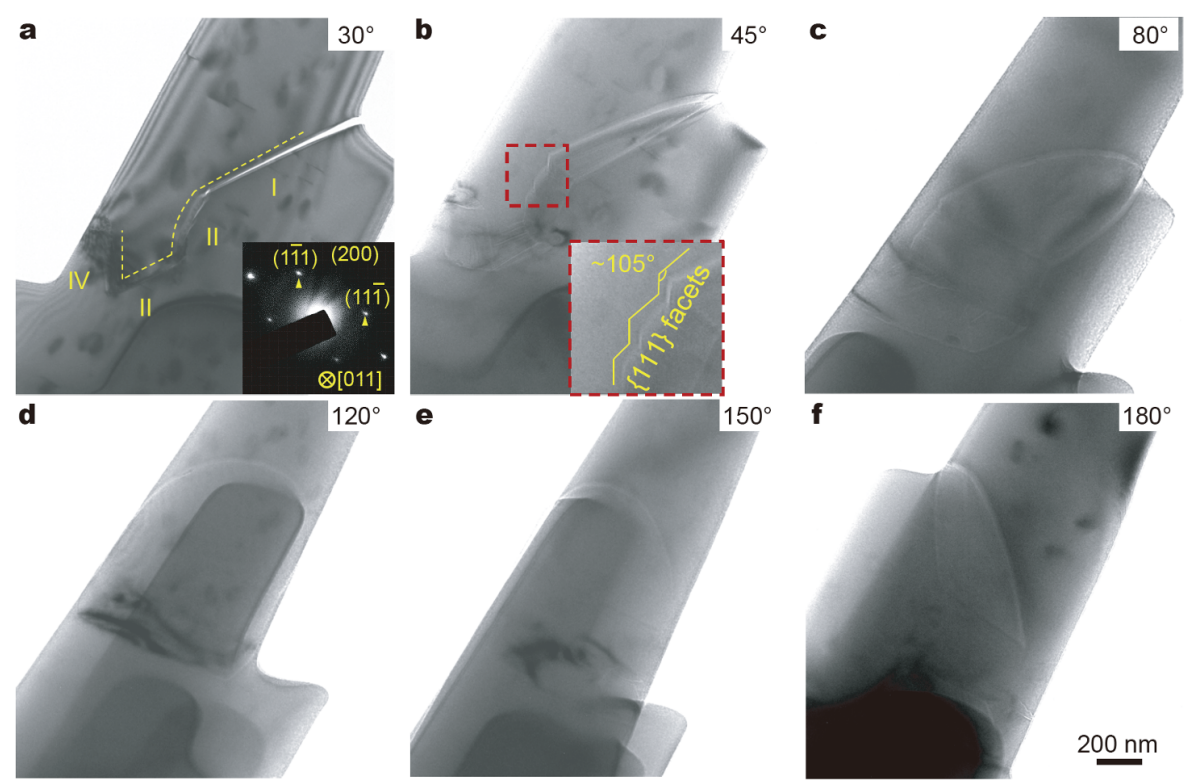

Figure $4(a-f)$ Crack morphology in a nanopillar viewed from different tilting angles. The inset in (a) shows the corresponding SAED pattern. The inset in (b) shows the magnified view of the boxed region.

$\sim 200 \mathrm{~nm}$, which caused a more localized strain. In this way, we could deliberately introduce a small notch resembling the indenter tip geometry. Benefiting from the highly localized high stress, a sharp crack nucleated at the center of the contact region. It propagated in a short distance to release the local strain energy without causing the failure of the whole sample. The crack was further driven forward by slowly increasing the load. The more detailed propagation process could be captured. As shown in an enlarged view in Fig. 5b, even if the crack was curved, it still consisted of a series of $\{111\}$ cleavage facets. Furthermore, we investigated the atomic structure of the crack tip region (Fig. $4 \mathrm{c}$ and d). Fig. $4 \mathrm{~d}$ shows that the atoms in the $\{111\}$ plane were dislocated, which meant that as the cracks propagated, lattice instabilities often occurred in the $\{111\}$ plane. The observed curved crack in the diamond nanoplates was consistent with the cleavage plane observed in the bending diamond nanoneedles. Both indicated that the fracture was dominated by the $\{111\}$ plane cleavage in the diamond.

\section{Comparison of the propensity of different cleavage modes} It is well known that diamond has the highest brittleness with several possible cleavage planes [28,29]. Our experimental investigations indicated that $\{111\}$ cleavage was the dominant failure mode under uniaxial tension along the $\langle 111\rangle,\langle 110\rangle$ and $\langle 100\rangle$ directions. The propensity of different cleavage modes could be compared
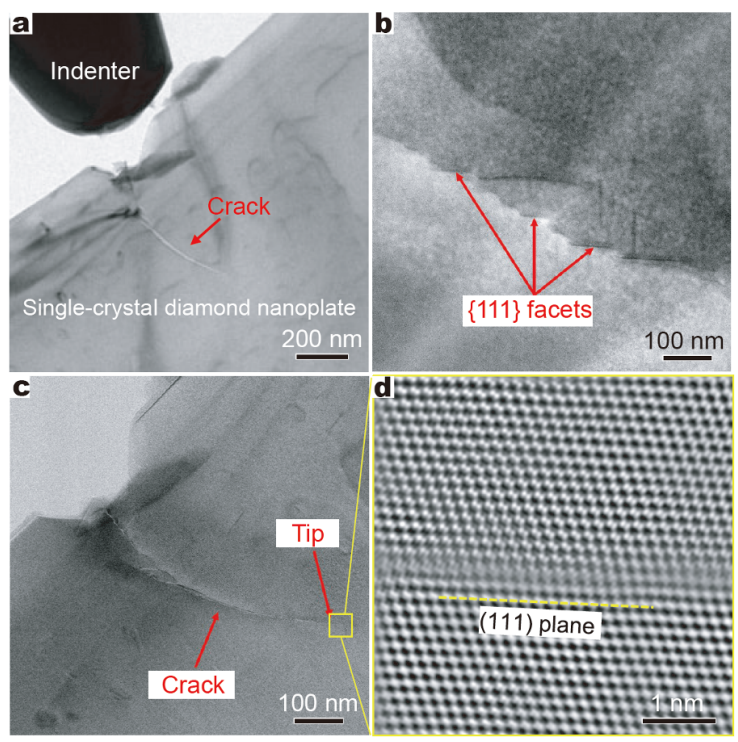

Figure 5 In situ cracking of single-crystal diamond nanoplate. (a) Indentation on a single-crystal diamond nano-plate. The crack was nucleated from the contact region. (b, c) Trace of the crack. (d) Atomic resolution characterization of the crack tip in the box in (c).

based on free energy consideration, with which the elastic instability could be probed at both the atomic and continuum levels $[30,31]$. Under a given deformation mode, the instability criterion could be derived by substituting the plane waveform displacement into the second-order 
derivative of free energy of a volume element:

$\Lambda(\boldsymbol{\omega}, \mathbf{n}) \equiv\left(C_{i j k l} \omega_{i} \omega_{k}+\tau_{j l}\right) n_{j} n_{l}$.

In the equation, $C_{i j k l}$ is the fourth-order elastic constant tensor, and $\tau_{j l}$ is the Cauchy stress tensor. Both $\omega_{i}$ and $n_{j}$ are the components of the unit vectors related to the waveform displacement in a Cartesian coordinate system, i.e., $\boldsymbol{\omega} \exp (\mathrm{i} n \cdot \boldsymbol{x})$, where $\boldsymbol{\omega}, \boldsymbol{n}$, and $\boldsymbol{x}$ represent the direction of the displacement, the norm of the invariant lattice plane, and the coordinate. The elastic instability mode was defined by the $(\boldsymbol{\omega}, \boldsymbol{n})$ pair. For the cleavage, $\boldsymbol{\omega}$ was parallel to the norm $\boldsymbol{n}$ of the cleavage plane. The condition for maintaining elastic stability is $\Lambda(\boldsymbol{\omega}, \boldsymbol{n})>0$, and the instability occurred when $\Lambda(\boldsymbol{\omega}, \boldsymbol{n})$ reached zero.

Fig. 6 a shows the stress-strain relationship calculated by first principles for a bulk diamond under uniaxial tension along the $\langle 111\rangle,\langle 110\rangle$ and $\langle 100\rangle$ directions. For large elastic deformations, the elastic constants no longer remained constant, as indicated by the nonlinearity in the stress-strain curves. In order to evaluate the $\Lambda(\boldsymbol{\omega}, \boldsymbol{n})$ criterion, the elastic constant tensor $C_{i j k l}$ was calculated for each configuration in the first-principle simulations with the uniaxial tension. The cleavage plane norms were chosen to be [111], [110], [100], [133], [122] and [233] in
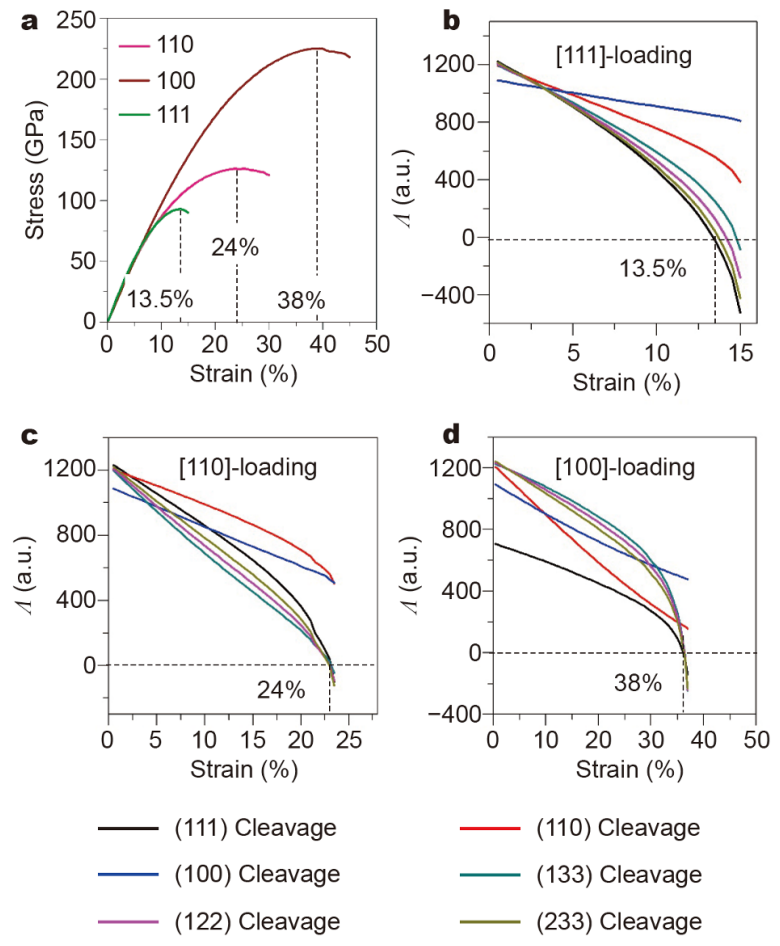

Figure 6 (a) Stress-strain curves under uniaxial tension along the $\langle 110\rangle$, $\langle 100\rangle$, and $\langle 111\rangle$ directions, calculated with first-principle simulations. (b-d) $\Lambda(\boldsymbol{\omega}, \boldsymbol{n})$ variation with the tensile strain for different lattice planes. order to compare the propensity of different cleavage modes. Clearly, all of the $\Lambda(\boldsymbol{\omega}, \boldsymbol{n})$ curves monotonically decreased with the tensile strain along all directions (Fig. 6b-d). The $\Lambda$-curves always dropped fastest, and they sharply approached zero for large tensile strains. The crossover occurred at the tensile strains of $13.5 \%$ (Fig. 6b), 24\% (Fig. 6c), and 38\% (Fig. 6d) along the $\langle 111\rangle$, $\langle 110\rangle$ and $\langle 100\rangle$ directions, respectively, which corresponded to the location of the stress maximum (Fig. 6a). The calculations showed that cleavage always occurred in the $\{111\}$ plane or the planes with orientations close to $\{111\}$. For example, it was difficult to tell whether $\{111\}$ cleavage or $\{233\}$ cleavage would occur because both $\Lambda$ criteria dropped to zero at approximately the same tensile strain, as shown in Fig. 6e. The small difference may have come from the error in the numerical simulations or some inaccuracy in modeling. However, our experimental investigation clearly showed the evidence that only $\{111\}$ cleavage occurred. This, however, provided complementary information for the theory.

As shown in Fig. 5d, the $\{111\}$ cleavage was characterized by the $\mathrm{C}-\mathrm{C}$ bond breakage at the atomic scale. The reason for this resided in the change of the $\mathrm{C}-\mathrm{C}$ bonds under deformation. Fig. 7 plots the electron density and the bond length change of the configurations for various tensile strains. For tension along the $\langle 111\rangle \mathrm{di}$ rection, the $\mathrm{C}-\mathrm{C}$ bonds perpendicular to the (111) plane had the largest bond length and the least electron density in between two neighboring $\mathrm{C}$ atoms. Therefore, this group of $\mathrm{C}-\mathrm{C}$ bonds was the most weakened and prone to breakage. The observation agreed with the above theoretical analysis. The trend was the same for the case of the $\langle 110\rangle$ tension, although the (111) plane was inclined to the loading direction. For tension along the $\langle 100\rangle$ direction, all of the $\mathrm{C}-\mathrm{C}$ bonds had the same response to the strain, indicating that all of the $\{111\}$ planes were possible cleavage planes.

\section{CONCLUSIONS}

The atomic-scale observation of the deformation and failure of diamonds was demonstrated by the X-Mech holder, which had a compact design of five-degree-offreedom actuation including both $\alpha$-tilt and $\beta$-tilt. The indenter was made of NTD. The durability was demonstrated by compressing the NTD pillar until continuous plastic flow occurred. To uncover the orientation-dependent fracture, bending tests were performed on the $\langle 111\rangle,\langle 110\rangle$ and $\langle 100\rangle$ single-crystal diamond needles. The $\{111\}$ cleavage was identified to be the dominant failure mode, which was consistent with the first-princi- 


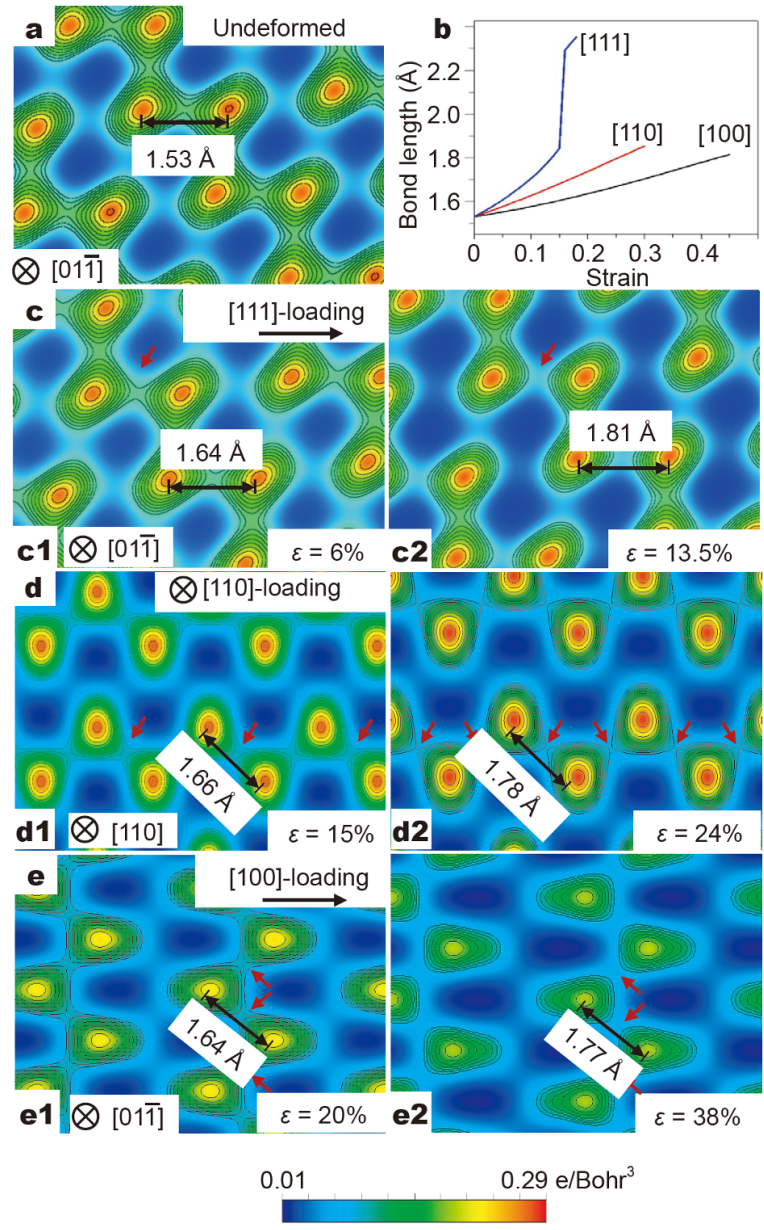

Figure 7 (a) Electron charge contour of the diamond in the (01) plane. (b) Maximum $\mathrm{C}-\mathrm{C}$ bond length versus strain under uniaxial tension along the [111], [110], and [100] directions. (c) Electron charge contour of the diamond under the [111]-direction tensile strains of $6 \%(\mathrm{c} 1)$ and $13.5 \%$ (c2) in the (011) plane. (d) Electron charge contour of the diamond under the [110]-direction tensile strains of $15 \%$ (d1) and $24 \%$ (d2) in the (110) plane. (e) Electron charge contour of the diamond under the [100]-direction tensile strains of $20 \%(\mathrm{~d} 1)$ and $38 \%(\mathrm{~d} 2)$ in the (011) plane. The maximum bond length is denoted in $(\mathrm{d}, \mathrm{e})$. The arrows point to the minimal electron charge density locations along the $\mathrm{C}-\mathrm{C}$ bonds.

ple simulations. The indentation on a diamond plate led to curved cracks consisting of nanometer-scale steps. Based on the atomic-scale observation, the steps could be clearly identified to be atomic flat $\{111\}$ facets. We expect that more delicate research can be carried out extensively by means of the X-Mech holder in the near future, including in-situ stimulation, atomic characterization, and tomography.

Received 7 February 2020; accepted 26 April 2020; published online 3 July 2020
1 Morishita S, Ishikawa R, Kohno Y, et al. Attainment of 40.5 pm spatial resolution using $300 \mathrm{kV}$ scanning transmission electron microscope equipped with fifth-order aberration corrector. Microscopy, 2017, 67: 46-50

2 Jia CL, Lentzen, M, Urban K. High-resolution transmission electron microscopy using negative spherical aberration. Microsc Microanal, 2004, 10: 174-184

3 O'Keefe MA, Hetherington CJD, Wang YC, et al. Sub-Ångstrom high-resolution transmission electron microscopy at $300 \mathrm{keV}$. Ultramicroscopy, 2001, 89: 215-241

4 Ketov SV, Ivanov YP, Şopu D, et al. High-resolution transmission electron microscopy investigation of diffusion in metallic glass multilayer films. Mater Today Adv, 2019, 1: 100004

5 Haque MA, Saif MTA. In-situ tensile testing of nano-scale specimens in SEM and TEM. Exp Mech, 2002, 42: 123-128

6 Spiecker E, Oh SH, Shan ZW, et al. Insights into fundamental deformation processes from advanced in situ transmission electron microscopy. MRS Bull, 2019, 44: 443-449

7 Nie A, Gan LY, Cheng Y, et al. Twin boundary-assisted lithium ion transport. Nano Lett, 2014, 15: 610-615

$8 \mathrm{Bu} \mathrm{Y,} \mathrm{Li} \mathrm{Z,} \mathrm{Liu} \mathrm{J,} \mathrm{et} \mathrm{al.} \mathrm{Nonbasal} \mathrm{slip} \mathrm{systems} \mathrm{enable} \mathrm{a} \mathrm{strong} \mathrm{and}$ ductile hexagonal-close-packed high-entropy phase. Phys Rev Lett, 2019, 122: 075502

9 Kacher J, Zhu T, Pierron O, et al. Integrating in situ TEM experiments and atomistic simulations for defect mechanics. Curr Opin Solid State Mater Sci, 2019, 23: 117-128

10 Nie A, Gan LY, Cheng Y, et al. Atomic-scale observation of lithiation reaction front in nanoscale $\mathrm{SnO}_{2}$ materials. ACS Nano, 2013, 7: 6203-6211

11 Legros M, Gianola DS, Motz C. Quantitative in situ mechanical testing in electron microscopes. MRS Bull, 2010, 35: 354-360

12 Nili $\mathrm{H}$, Kalantar-zadeh $\mathrm{K}$, Bhaskaran $\mathrm{M}$, et al. In situ nanoindentation: Probing nanoscale multifunctionality. Prog Mater Sci, 2013, 58: 1-29

13 Bernal RA, Ramachandramoorthy R, Espinosa HD. Double-tilt in situ TEM holder with multiple electrical contacts and its application in MEMS-based mechanical testing of nanomaterials. Ultramicroscopy, 2015, 156: 23-28

14 Shi Z, Tsymbalov E, Dao M, et al. Deep elastic strain engineering of bandgap through machine learning. Proc Natl Acad Sci USA, 2019, 116: 4117-4122

15 Watanabe H, Nebel CE, Shikata S. Isotopic homojunction band engineering from diamond. Science, 2009, 324: 1425-1428

16 Isberg J. High carrier mobility in single-crystal plasma-deposited diamond. Science, 2002, 297: 1670-1672

17 May PW. Materials science: The new diamond age? Science, 2008, 319: 1490-1491

18 Nie A, Bu Y, Li P, et al. Approaching diamond's theoretical elasticity and strength limits. Nat Commun, 2019, 10: 5533

19 Banerjee A, Bernoulli D, Zhang $\mathrm{H}$, et al. Ultralarge elastic deformation of nanoscale diamond. Science, 2018, 360: 300-302

20 Zhang Y, Bu Y, Fang X, et al. A compact design of four-degree-offreedom transmission electron microscope holder for quasi-fourdimensional characterization. arxiv: 2001.07074

21 Huang Q, Yu D, Xu B, et al. Nanotwinned diamond with unprecedented hardness and stability. Nature, 2014, 510: 250-253

22 Rappe AM, Rabe KM, Kaxiras E, et al. Optimized pseudopotentials. Phys Rev B, 1990, 41: 1227-1230

23 Monkhorst HJ, Pack JD. Special points for brillouin-zone integrations. Phys Rev B, 1976, 13: 5188-5192 
24 Nie A, Bu Y, Huang J, et al. Direct observation of room-temperature dislocation plasticity in diamond. Matter, 2020, 2: 12221232

$25 \mathrm{Bu} \mathrm{Y,} \mathrm{Wang} \mathrm{P,} \mathrm{Nie} \mathrm{A,} \mathrm{et} \mathrm{al.} \mathrm{Dislocation} \mathrm{slip} \mathrm{or} \mathrm{phase} \mathrm{transfor-}$ mation lead to room-temperature plasticity in diamond: Comment on plastic deformation of single-crystal diamond nanopillars. arxiv: 2002.01104

26 Liu C, Song X, Li Q, et al. Smooth flow in diamond: Atomistic ductility and electronic conductivity. Phys Rev Lett, 2019, 123: 195504

$27 \mathrm{Hu} \mathrm{W}$, Wen B, Huang Q, et al. Role of plastic deformation in tailoring ultrafine microstructure in nanotwinned diamond for enhanced hardness. Sci China Mater, 2017, 60: 178-185

28 Bassett WA, Skalwold EA. Diamond cleavage: Importance to high pressure research. High Pressure Res, 2017, 37: 46-58

29 Field JE. The mechanical and strength properties of diamond. Rep Prog Phys, 2012, 75: 126505

30 Li J, Van Vliet KJ, Zhu T, et al. Atomistic mechanisms governing elastic limit and incipient plasticity in crystals. Nature, 2002, 418: 307-310

31 Li J, Zhu T, Yip S, et al. Elastic criterion for dislocation nucleation. Mater Sci Eng-A, 2004, 365: 25-30

Acknowledgements This work was supported by the National Natural Science Foundation of China (11725210, 11672355 and 11702165), and the National Key R\&D Program of China (2018YFA0703400).

Author contributions Tian $\mathrm{Y}$ and Wang $\mathrm{H}$ initiated the project and created the experimental protocols. Nie A and Wang $\mathrm{H}$ designed the special TEM holder. Zhang $\mathrm{Y}$ assembled and tested the entire setup. Huang $\mathrm{J}$ and Jin $\mathrm{T}$ carried out the fabrication of the diamond nanopillars. Bu Y and Nie A conducted the in-situ TEM testing and corresponding data analysis. Nie $\mathrm{A}$, Wang $\mathrm{H}$, and Tian $\mathrm{Y}$ wrote the manuscript, and all of the authors contributed to the discussion and revision of the manuscript.

Conflict of interest The authors declare that they have no conflict of interest.

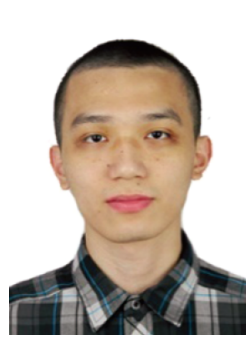

Yizhi Zhang is a $\mathrm{PhD}$ candidate at the Center for X-Mechanics, Zhejiang University, China. His research interest focuses on instrumental development for in-situ transmission electron microscopy and its applications on micro- and nanomechanics, including the X-Nano system.

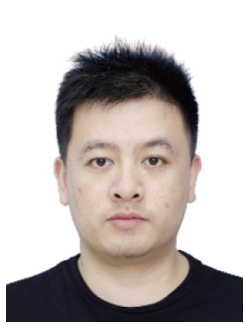

Yeqiang Bu is a $\mathrm{PhD}$ candidate at the Center for X-Mechanics, Zhejiang University, China. His current research interest focuses on employing the state-of-art in-situ transmission electron microscopy technique to study the mechanical properties and deformation behavior of superhard materials (e.g., diamond and cubic boron nitride).

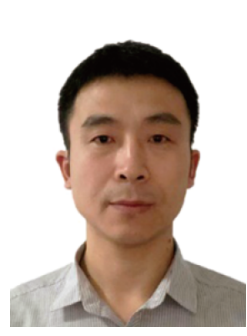

Anmin Nie is a professor at the Center for High Pressure Science, State Key Laboratory of Metastable Materials Science and Technology, Yanshan University. He received his $\mathrm{PhD}$ degree in solid mechanics from Zhejiang University in 2012. During 2012-2016, he worked at Michigan Technological University and the University of Illinois at Chicago as a postdoctoral research associate. His research interests are in situ microscopy on nano-scaled materials and their behaviors.

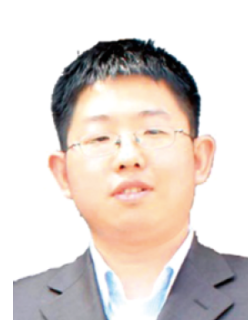

Hongtao Wang is a professor at Zhejiang University. His research interests focus on the development of instruments for the interdisciplinary research in the fields of mechanics and material science. He has developed a 4-degree-freedom nano manipulator dedicated to both TEM tomography and in-situ TEM. A combination of the two methods can promote deeper understanding of microstructural evolution in $3 \mathrm{D}$.

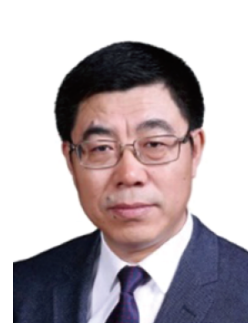

Yongjun Tian is a professor at the Center for High Pressure Science, State Key Laboratory of Metastable Materials Science and Technology, Yanshan University. He received his $\mathrm{PhD}$ degree from the Institute of Physics, Chinese Academy of Sciences, in 1994, and worked as a postdoctoral fellow at the Universität Jena supported by Humboldt Research Fellowships from 1996 to 1998. His research interests include the design and synthesis of novel metastable materials.

\section{双倾原位透射电镜力学样品台的设计及原子尺度 下对金刚石变形和断裂的观察}

张奕志 ${ }^{1,2 \dagger}$, 卜叶强 ${ }^{1 \dagger}$, 黄俊全 ${ }^{3}$, 靳天野 $^{3}$, 聂安民 $^{3^{*}}$, 王宏涛 ${ }^{1,2^{*}}$, 田永君

摘要 原位透射电子显微技术 (in-situ TEM) 可以动态观察材料内 部缺陷的交互作用, 是研究材料构效关系的重要手段. 本文报道了 一种自主研制的力学加载双倾in-situ TEM样品台, 这种样品台具 有前后、左右、上下、旋转以及 $\beta$ 倾转五个维度的操控参数, 并结 合了世界上最硬的纳米孪晶金刚石制作压头，可实现在极高载荷 下样品的高分辨原位观察, 并可对样品进行 $360^{\circ}$ 三维空间重构. 利 用该样品台，对 $\langle 100\rangle ，\langle 110\rangle$ 及 $\langle 111\rangle$ 取向的金刚石纳米针进行了原 位弯曲, 发现金刚石纳米针可实现大的弯曲变形, 并最终沿 $\{111\}$ 解 理断裂. 同时利用该样品台对金刚石纳米片进行了原位压痕实验, 在原子尺度下观察了金刚石纳米片中裂纹的扩展, 发现裂纹由原 子级平整的 $\{111\}$ 面台阶构成. 具有超高强度和硬度金刚石的变形 和屈服以及相应原子尺度下的原位表征证实了该in-situ TEM样品 台的稳定性和可靠性, 未来基于该样品台可拓展更广泛的研究, 包 括材料的多场加载、原子尺度原位表征及三维成像. 